Pacific

Journal of

Mathematics

\title{
ON THE UNIT GROUP OF SOME MULTIQUADRATIC NUMBER FIELDS
}

Elliot Benjamin, Franz Lemmermeyer AND Chip SNyder 


\title{
ON THE UNIT GROUP OF SOME MULTIQUADRATIC NUMBER FIELDS
}

\author{
Elliot Benjamin, Franz Lemmermeyer And Chip SNyder
}

\begin{abstract}
We study the index of the group of units in the genus field of an imaginary quadratic number field modulo the subgroup generated by the units of the quadratic subfields (over $\mathbb{Q}$ ) of the genus field.
\end{abstract}

\section{Introduction}

One major problem in algebraic number theory is the computation of the class number $h(K)$ for a number field $K$. In the case of quadratic fields, this problem is easily solved by elementary methods. Once the field degree is larger than 2 , the problem becomes more challenging. Historically, the oldest case after the quadratic fields seems to be when $K$ runs through a particular family of quartic bicyclic fields over $\mathbb{Q}$, meaning that $\operatorname{Gal}(K / \mathbb{Q}) \simeq(2,2)$ (here $\left(a_{1}, \ldots, a_{r}\right)$ denotes the direct sum of cyclic groups of order $a_{i}$, for $\left.i=1, \ldots, r\right)$. Dirichlet [1842] in essence computed the class number $h(K)$ for the family of quartic fields $K=\mathbb{Q}(\sqrt{-1}, \sqrt{m}), m$ a positive nonsquare integer. Namely, let $k_{1}=\mathbb{Q}(\sqrt{-1}), k_{2}=\mathbb{Q}(\sqrt{m})$, and $k_{3}=$ $\mathbb{Q}(\sqrt{-m})$, and denote by $E_{F}$ the group of units of a number field $F$. Then Dirichlet discovered the class number formula

$$
h(K)=\frac{1}{2} q(K / \mathbb{Q}) h\left(k_{2}\right) h\left(k_{3}\right),
$$

where $q=q(K / \mathbb{Q})=\left(E_{K}: E_{k_{1}} E_{k_{2}} E_{k_{3}}\right)$. Dirichlet went on to show that the unit index $q$ could be determined and was equal to 1 or 2 .

Over time, Dirichlet's formula has been generalized in several directions; see in particular [Herglotz 1922; Kubota 1953; 1956; Kuroda 1950; Lemmermeyer 1994b; Wada 1966], and references therein. One particularly striking formula is usually attributed to Kuroda [1950], but in fact goes back to Herglotz [1922] in an equivalent, if less convenient, form for $q$. Let $L=\prod_{i} k_{i}$ be the multiquadratic field generated as the composite of all its quadratic subfields $k_{i}$, and suppose further that $[L: \mathbb{Q}]=2^{m}$. Then

$$
h(L)=\frac{1}{2^{v}} q(L / \mathbb{Q}) \prod_{i} h\left(k_{i}\right),
$$

MSC2000: primary 11R11; secondary 11R27, 11R29.

Keywords: unit index, Kuroda's class number formula, multiquadratic fields. 
where $q=q(L / \mathbb{Q})=\left(E_{L}: \prod_{i} E_{k_{i}}\right)$ and

$$
v= \begin{cases}m\left(2^{m-1}-1\right) & \text { if } L \text { is real } \\ (m-1)\left(2^{m-2}-1\right)+2^{m-1}-1 & \text { if } L \text { is complex. }\end{cases}
$$

Hence $h(L)$ can be computed easily provided that the unit index $q(L / \mathbb{Q})$ can be computed. Herein lies the obstruction to an easy determination of the class number of multiquadratic number fields. For quartic bicyclic fields, Kubota [1956] gave a method for finding a system of fundamental units and thus for computing $q$. Wada [1966], generalized Kubota's method, creating an algorithm for computing fundamental units in any given multiquadratic field. However, in general there seems to be no explicit formula for $q$, even when $L$ is of degree 4 over $\mathbb{Q}$.

This brings us to the purpose of this article. We try to glean some understanding of the difficulties in computing the unit index by giving explicit computations of $q$ for special families of multiquadratic fields $L$. We consider the special case of the genus field $L=k_{\text {gen }}$ of a complex quadratic field $k$ for which the 2-rank of the class group $\mathrm{Cl}(k)$ of $k$ is $\leq 3$. (Recall that the $2^{n}$-rank of a finite abelian group $G$ is the minimal number of generators of the factor group $G^{2^{n-1}} / G^{2^{n}}$.) If the 2-rank of $\mathrm{Cl}(k)$ is 1 , then $[L: \mathbb{Q}]=4$, by genus theory, and in this case it is known that $q=1$ (see [Lemmermeyer 1995], for instance; the proof is easy - see the next section).

Next, if the 2-rank is 2 , then $[L: \mathbb{Q}]=8$ by genus theory. In this case, we reduce the problem to that of computing $q(K / \mathbb{Q})$ where $K$ is the maximal real subfield of $L$. But then $K$ is a totally real bicyclic field and we may apply the results of [Kubota 1956] to compute $q(K / \mathbb{Q})$. We find that $q(L / \mathbb{Q})=8$ or 2 according as the 2-class field tower of $k$ is of length 1 or $>1$. (Here $k^{1}$ is the Hilbert 2-class field of $k$ and $k^{n+1}=\left(k^{n}\right)^{1}$; the length of the 2-class field tower of $k$ is the cardinality of the set of $k^{n}$.)

For the case where the 2-rank of $\mathrm{Cl}(k)$ is 3 , we seem to be in new territory. We restrict to the case of elementary 2-class group. Specifically, we assume $\mathrm{Cl}_{2}(k) \simeq$ $(2,2,2)$, so $L=k_{\text {gen }}=k^{1}$. If the rank of $\mathrm{Cl}_{2}\left(k^{1}\right)$ is 2 as a module over the integral group ring $\Lambda=\mathbb{Z}\left[\mathrm{Gal}\left(k^{1} / k\right)\right]$, then $q(L / \mathbb{Q})=2^{7}$. This condition on the $\Lambda$-rank is, by the way, a natural one; see [Benjamin et al. 2003]. We then obtain less complete information about $q$ for the other case where $\mathrm{Cl}_{2}\left(k^{1}\right)$ is of $\Lambda$-rank 3. In the particular fields we consider, $q=2^{4}$ or $2^{5}$.

\section{The Main Results}

Let $k$ be an imaginary quadratic field for which the 2-rank of $\mathrm{Cl}(k)$ is $t-1$. Hence, by genus theory, $k=\mathbb{Q}\left(\sqrt{d_{1} \cdots d_{t}}\right)$, where disc $k=d_{1} \cdots d_{t}$ is a factorization of the discriminant of $k$ into distinct prime discriminants $d_{i}$ divisible by the rational prime $p_{i}$ for $i=1, \ldots, t$. Then $L=k_{\mathrm{gen}}=\mathbb{Q}\left(\sqrt{d_{1}}, \ldots, \sqrt{d_{t}}\right)$ and hence multiquadratic 
of degree $2^{t}$ over $\mathbb{Q}$. Hence for $t \geq 2$ Kuroda's class number formula above yields

$$
h(L)=\frac{1}{2^{v}} q(L / \mathbb{Q}) \prod_{i} h\left(k_{i}\right)
$$

where $k_{i}$ range over the $2^{t}-1$ quadratic subfields of $L, q(L / \mathbb{Q})=\left(E_{L}: \prod_{i} E_{k_{i}}\right)$, and $v=(t-1)\left(2^{t-2}-1\right)+2^{t-1}-1$ since $L$ is complex.

We start our computations of $q=q(L / \mathbb{Q})$ by first considering $t=2$ (for the sake of completeness).

Theorem 1. Let $k$ be a complex quadratic number field and $L=k_{\mathrm{gen}}$. If the 2-rank of $\mathrm{Cl}(k)$ equals 1 , then $q(L / \mathbb{Q})=1$.

Proof. Since the 2-rank of $\mathrm{Cl}(k)$ is $1, t=2$ so $k=\mathbb{Q}\left(\sqrt{d_{1} d_{2}}\right)$ for prime discriminants $d_{1}, d_{2}$. Then $L=\mathbb{Q}\left(\sqrt{d_{1}}, \sqrt{d_{2}}\right)$. Now, by Kuroda's class number formula (where $t=2$ implies $v=1$ ),

$$
h(L)=\frac{1}{2} q(L / \mathbb{Q}) h\left(d_{1} d_{2}\right) h\left(d_{1}\right) h\left(d_{2}\right),
$$

where $h(n)=h(\mathbb{Q}(\sqrt{n}))$. Now, it is well known that $h(d)$ is odd for any prime discriminant $d$. Moreover, by the Artin map, $\operatorname{Gal}\left(k^{1} / k\right) \simeq \mathrm{Cl}_{2}(k)$ where $\mathrm{Cl}_{2}(k)$ is the 2-class group of $k$, the Sylow 2-subgroup of the class group, the order of which is $h_{2}(k)$, the 2-class number of $k$. Now consider $G=\operatorname{Gal}\left(k^{2} / k\right)$. Since the commutator subgroup $G^{\prime}=\operatorname{Gal}\left(k^{2} / k^{1}\right)$, we see $G / G^{\prime} \simeq \operatorname{Gal}\left(k^{1} / k\right) \simeq \mathrm{Cl}_{2}(k)$. But in the present case, $\mathrm{Cl}_{2}(k)$ is cyclic, whence $G^{\prime}=\langle 1\rangle$, and thus $k^{2}=k^{1}$. But then since in general $k^{1} \subseteq L^{1} \subseteq k^{2}$, we have $L^{1}=k^{1}$. Therefore, $h_{2}(L)=\left[L^{1}: L\right]=$ $\left[k^{1}: L\right]=\left[k^{1}: k\right] / 2=h_{2}(k) / 2$. Now by restricting to 2 -class numbers and using the fact that $q$ is a power of two, (see [Wada 1966], for instance) the Kuroda class number formula becomes

$$
h_{2}(L)=\frac{1}{2} q(L / \mathbb{Q}) h_{2}(k) h_{2}\left(d_{1}\right) h_{2}\left(d_{2}\right) .
$$

From the preceding discussion we get $\frac{1}{2} h_{2}(k)=\frac{1}{2} q h_{2}(k)$, as needed.

Next, we consider the case where the 2-rank of $\mathrm{Cl}(k)$ is 2, i.e. $t=3$. Hence $k=\mathbb{Q}\left(\sqrt{d_{1} d_{2} d_{3}}\right)$, with prime discriminants $d_{i}$. Moreover, since $k$ is complex, disc $k<0$ so either all the $d_{i}$ are negative or exactly two are positive, say $d_{1}, d_{2}>0$, $d_{3}<0$. Notice that we have $L=\mathbb{Q}\left(\sqrt{d_{1}}, \sqrt{d_{2}}, \sqrt{d_{3}}\right)$. Let $K=L^{+}$be the maximal real subfield of $L$, (so $K=\mathbb{Q}\left(\sqrt{d_{1}}, \sqrt{d_{2}}\right.$ ) if say $d_{1}, d_{2}>0, d_{3}<0$, and $K=\mathbb{Q}\left(\sqrt{d_{1} d_{2}}, \sqrt{d_{2} d_{3}}\right)$ if $d_{i}<0$, for $\left.i=1,2,3\right)$. But then it follows that $q(L / \mathbb{Q})=Q(L / K) q(K / \mathbb{Q})$, where $Q(L / K)=\left(E_{L}: W_{L} E_{K}\right)$ with $W_{L}$ the group of roots of unity of $L$. To see this apply for example [Benjamin et al. 2003, Proposition 1], where we notice that any primitive eighth $\operatorname{root} \zeta_{8}$ of unity is not contained in $L$ since any ramification index of a prime in $L / \mathbb{Q}$ must divide 2 , whereas 2 is totally ramified in $\mathbb{Q}\left(\zeta_{8}\right)$. Now suppose $d_{1}, d_{2}>0, d_{3}<0$. By [Lemmermeyer 
1995, Theorem 1], $Q(L / K)=1$, since $L=K\left(\sqrt{d_{3}}\right)$ implies that $L / K$ is essentially ramified if $d_{3} \neq-4$ and that $20_{K}$ is not an ideal square if $d_{3}=-4$, see [Lemmermeyer 1995] again for the details. Thus we have $q(L / \mathbb{Q})=q(K / \mathbb{Q})$. If however all the $d_{i}<0$, then for any $i, L=K\left(\sqrt{d_{i}}\right)$. In this case, it can be shown that $Q(L / K)=2$ by [Lemmermeyer 1995], but we shall see that this is the case by another method. In either case, it is well known that $Q(L / K)=1,2$ (see [Hasse 1985 , Satz 14]), and moreover, by Kubota [Kubota 1956], $q(K / \mathbb{Q})$ divides 4 . Thus $q(L / \mathbb{Q})$ must divide 8 .

Theorem 2. Let $k$ be a complex quadratic field with 2-rank $\mathrm{Cl}(k)=2$. Then for $L=k_{\mathrm{gen}}, q(L / \mathbb{Q})=8$ or 2 according as the 2-class field tower of $k$ is of length 1 or $>1$.

Proof. By assumption, $k=\mathbb{Q}\left(\sqrt{d_{1} d_{2} d_{3}}\right)$ for prime discriminants $d_{i}$. Now notice that $K_{i}=k\left(\sqrt{d_{i}}\right)$ for $i=1,2,3$ are the three unramified quadratic extensions of $k$ in $L$. These fields are quartic bicyclic extensions of $\mathbb{Q}$ and so Kuroda's class number yields

$$
h\left(K_{1}\right)=\frac{1}{2} q\left(K_{1} / \mathbb{Q}\right) h(k) h\left(d_{2} d_{3}\right) h\left(d_{1}\right),
$$

since $m=2$, so $v=1$, (analogously for $K_{2}$ and $K_{3}$ ). Now since the $K_{i}$ are unramified quadratic extensions of a complex quadratic field $k$, it is known that $q\left(K_{i} / \mathbb{Q}\right)=1$; see for example [Lemmermeyer 1995]. Hence by considering 2class numbers so that we may use $h_{2}\left(d_{i}\right)=1$, we have

$h_{2}(L)=\frac{1}{32} q(L / \mathbb{Q}) h_{2}(k) h_{2}\left(d_{1} d_{2}\right) h_{2}\left(d_{1} d_{3}\right) h_{2}\left(d_{2} d_{3}\right), \quad h_{2}\left(K_{1}\right)=\frac{1}{2} h_{2}(k) h_{2}\left(d_{2} d_{3}\right)$.

Now we rewrite the formula for $h_{2}(L)$ in terms of $h_{2}\left(K_{i}\right)$. From above, notice that for example $h_{2}\left(d_{2} d_{3}\right)=2 h_{2}\left(K_{1}\right) / h_{2}(k)$, etc. and so by class field theory,

$$
h_{2}\left(d_{2} d_{3}\right)=\frac{2\left[K_{1}^{1}: K_{1}\right]}{\left[k^{1}: k\right]}=\frac{2\left[K_{1}^{1}: k^{1}\right]\left[k^{1}: K_{1}\right]}{\left[k^{1}: K_{1}\right]\left[K_{1}: k\right]}=\left[K_{1}^{1}: k^{1}\right] .
$$

Substituting into the above formula yields

$$
\left[L^{1}: L\right]=\frac{1}{32} q(L / \mathbb{Q})\left[k^{1}: k\right]\left[K_{1}^{1}: k^{1}\right]\left[K_{2}^{1}: k^{1}\right]\left[K_{3}^{1}: k^{1}\right]
$$

and since $[L: k]=4$, we have

$$
\left[L^{1}: k^{1}\right]=\frac{1}{8} q(L / \mathbb{Q})\left[K_{1}^{1}: k^{1}\right]\left[K_{2}^{1}: k^{1}\right]\left[K_{3}^{1}: k^{1}\right] .
$$

Notice, in particular, that if the 2-class field tower of $k$ is of length 1, then all the field degrees in the above formula equal 1 , and therefore $q=8$. Now, the length of the 2-class field tower of $k$ is 1 precisely when $d_{i}<0$ for $i=1,2,3$; see for example [Benjamin et al. 1997]. From this we have $8=q(L / \mathbb{Q})=Q(L / K) q(K / \mathbb{Q})$ from which it follows (by the comments before the proposition) that $Q(L / K)=2$ and $q(K / \mathbb{Q})=4$. 
Now suppose that $d_{1}, d_{2}>0, d_{3}<0$. In this case we have $q(L / \mathbb{Q})=q(K / \mathbb{Q})$, where $K=\mathbb{Q}\left(\sqrt{d_{1}}, \sqrt{d_{2}}\right)$. Kuroda's class number formula implies

$$
h_{2}(K)=\frac{1}{4} q(K / \mathbb{Q}) h_{2}(F),
$$

where $F=\mathbb{Q}\left(\sqrt{d_{1} d_{2}}\right)$. Then notice that $\mathrm{Cl}_{2}(F)$ is cyclic and thus $F^{1}=F^{2}$. Thus since $K / F$ is unramified so $K \subseteq F^{1}, h_{2}(K)=h_{2}(F) / 2$. Plugging this into the formula above yields $q(K / \mathbb{Q})=2$. Thus $q(L / \mathbb{Q})=2$.

Proposition 3. Let $k$ be a complex quadratic field with 2-rank $\mathrm{Cl}(k) \leq 2$ and with 4-rank $\mathrm{Cl}(k) \leq 1$. Then

$$
\prod_{i} K_{i}^{1}=\left(\prod_{i} K_{i}\right)^{1}
$$

where $K_{i}$ range over all the unramified quadratic extensions of $k$.

Proof. If $k^{1}=k^{2}$, then the proposition is trivially true, since both fields are $k^{1}$. Thus, assume $k^{1} \neq k^{2}$. Hence we know $k=\mathbb{Q}\left(\sqrt{d_{1} d_{2} d_{3}}\right)$ where $d_{1}, d_{2}>0, d_{3}<0$. From the proof of Theorem 2,

$$
\left[L^{1}: k^{1}\right]=\frac{1}{4}\left[K_{1}^{1}: k^{1}\right]\left[K_{2}^{1}: k^{1}\right]\left[K_{3}^{1}: k^{1}\right],
$$

where $L=K_{1} K_{2} K_{3}$ with $K_{i}=k\left(\sqrt{d_{i}}\right)$. But notice that

$$
\left[K_{1}^{1} K_{2}^{1} K_{3}^{1}: k^{1}\right]=\frac{\left[K_{1}^{1}: k^{1}\right]}{\left[K_{1}^{1} \cap K_{2}^{1} K_{3}^{1}: k^{1}\right]} \frac{\left[K_{2}^{1}: k^{1}\right]}{\left[K_{2}^{1} \cap K_{3}^{1}: k^{1}\right]}\left[K_{3}^{1}: k^{1}\right] .
$$

(Also notice this equation is true for any permutation of the indices.) Now since

$$
\left[L^{1}: k^{1}\right]=\left[L^{1}: K_{1}^{1} K_{2}^{1} K_{3}^{1}\right]\left[K_{1}^{1} K_{2}^{1} K_{3}^{1}: k^{1}\right],
$$

we see by putting these equations together that

$$
\left[L^{1}: K_{1}^{1} K_{2}^{1} K_{3}^{1}\right]=\frac{1}{4}\left[K_{1}^{1} \cap K_{2}^{1} K_{3}^{1}: k^{1}\right]\left[K_{2}^{1} \cap K_{3}^{1}: k^{1}\right] .
$$

To finish the proof, it suffices to show that

$$
\left[K_{1}^{1} \cap K_{2}^{1} K_{3}^{1}: k^{1}\right]=\left[K_{2}^{1} \cap K_{3}^{1}: k^{1}\right]=2 .
$$

Here is where some group theory comes in. Let $G=\operatorname{Gal}\left(k^{2} / k\right)$, and further let $H_{1}, H_{2}, H_{3}$ be the three maximal subgroups of $G$ such that $\operatorname{Gal}\left(k^{2} / K_{i}\right)=H_{i}$. Then we need to show that

$$
\left(G^{\prime}: H_{2}^{\prime} H_{3}^{\prime}\right)=\left(G^{\prime}: H_{1}^{\prime}\left(H_{2}^{\prime} \cap H_{3}^{\prime}\right)\right)=2 .
$$

Here is a sketch of the proof. If $G^{\prime}$ is cyclic, say $G^{\prime}=\langle c\rangle$, by the table of possible groups and their presentations at the end of [Benjamin et al. 1997], we have (without loss of generality) $H_{3}^{\prime}=\left\langle c^{2}\right\rangle$ and $H_{1}^{\prime} H_{2}^{\prime}=\left\langle c^{2}\right\rangle$, from which our result follows. 
Now suppose $G^{\prime}$ is not cyclic. Then by our assumption on the class group of $k$, $G$ must be nonmetacyclic with $G / G^{\prime} \simeq\left(2,2^{n}\right)$ for some $n>1$. Now we assume the notation before [Benjamin et al. 2001, Lemma 1]. Hence let $G=\langle a, b\rangle$ where $a^{2} \equiv$ $b^{2^{n}} \equiv 1 \bmod G^{\prime}$. Let $[a, b]=c$ and define inductively, $c_{2}=c$ and $c_{j+1}=\left[b, c_{j}\right]$. We have $G^{\prime}=\left\langle c_{2}, c_{3}, \ldots\right\rangle$, and $G_{3}=\left\langle c_{2}^{2}, c_{3}, \ldots\right\rangle$, and $G_{4}=\left\langle c_{2}^{4}, c_{3}^{2}, c_{4}, \ldots\right\rangle$; see [Benjamin et al. 1997, Lemma 2]. Now if $H_{3}=\left\langle a, b^{2}, G^{\prime}\right\rangle$, then it is easy to see that $H_{3}^{\prime} G_{4}=G_{3}$. Thus $H_{3}^{\prime}=G_{3}$ by [Hall 1933, Theorem 2.49ii]. Hence $\left(G^{\prime}: H_{3}^{\prime}\right)=\left(G^{\prime}: G_{3}\right)=2$. Similarly, if $H_{1}=\left\langle b, G^{\prime}\right\rangle$ and $H_{2}=\left\langle a b, G^{\prime}\right\rangle$, then $H_{1}^{\prime} H_{2}^{\prime} G_{4}=G_{3}$ so once again $H_{1}^{\prime} H_{2}^{\prime}=G_{3}$. This shows the result and finishes the proof of the proposition.

Now we consider $\mathrm{Cl}_{2}(k) \simeq(2,2,2)$, and thus in particular disc $k=d_{1} d_{2} d_{3} d_{4}$ for distinct prime discriminants $d_{i}$. If we assume the $\Lambda$-rank of $\mathrm{Cl}_{2}\left(k^{1} / k\right)$ is 2 , then by [Benjamin et al. 2003, Theorem 2], exactly three of the $d_{i}$ 's must be negative, say $d_{1}, d_{2}, d_{3}<0, d_{4}>0$.

Theorem 4. Let $k$ be a complex quadratic field with $\mathrm{Cl}_{2}(k) \simeq(2,2,2)$. If the $\Lambda$-rank of $\mathrm{Cl}_{2}\left(k^{1}\right)$ equals 2 , the unit index $q\left(k^{1} / \mathbb{Q}\right)$ equals $2^{7}$.

Proof. If $\mathrm{Cl}_{2}(k) \simeq(2,2,2)$, then $\mathrm{Cl}_{2}\left(k^{1} / k\right)$ has $\Lambda$-rank 2 if and only if $G / G^{\prime} \simeq$ $(2,2,2)$ and $G^{\prime} / G_{3} \simeq(2,2)$, where $G=\operatorname{Gal}\left(k^{2} / k\right)$. Thus $\left(G: G_{3}\right)=32$ and $G^{\prime} / G_{3} \simeq(2,2)$, and by [Hall and Senior 1964], $G / G_{3}$ must be one of the seven groups 32.033 , 32.035, 32.036, 32.037, 32.038, 32.040, 32.041, in the notation of that same reference.

Let $L=k^{1}=k_{\text {gen }}$. Kuroda's class number formula (with $t=4$, so $v=16$ ) gives

$$
h_{2}(L)=\frac{1}{2^{16}} q(L / \mathbb{Q}) h_{2}(k) \prod_{i} h_{2}\left(k_{i}\right),
$$

where the $k_{i}$ are the quadratic subfields of $L$ excluding $k$.

The following table lists the 2-class numbers $h_{2}\left(k_{i}\right)$ and $h_{2}(L)$ :

\begin{tabular}{l|l|l}
$G / G_{3}$ & $h_{2}\left(k_{i}\right)$ & $h_{2}(L)$ \\
\hline $32.041,32.040$ & $1(7), 2(6), 4$ & 4 \\
$32.035,32.037,32.038$ & $1(7), 2(5), 4,2^{n}$ & $2^{n+1}$ \\
32.036 & $1(7), 2(5), 2^{m+1}, 2^{n}$ & $2^{m+n}$ \\
32.033 & $1(7), 2(3), 4,2^{l}, 2^{m}, 2^{n}$ & $2^{l+m+n-1}$
\end{tabular}

Here "1 (7)" means that 7 quadratic subfields have 2-class number equal to 1 . Plugging these data into (1) we immediately find the values of the unit index $q(L / \mathbb{Q})$ in each of the cases.

The 2-class numbers of the quadratic subfields $k_{i}$ of $L$ are easily determined using genus theory (see [Kaplan 1976], for instance). The 2-class numbers of $L$ 
were computed in [Benjamin et al. 2003], except for the group $G / G_{3}=32.033$ (for the first five groups, we have given the structure of $G^{\prime}$ explicitly; in the case $G / G_{3}=32.036$, we computed the 2-class number and actually showed that $\left.q(L / \mathbb{Q})=2^{7}\right)$.

We will now study the case $G / G_{3} \simeq 32.033$ in detail. By Proposition 16 in the same reference, we have $k=\mathbb{Q}\left(\sqrt{d_{1} d_{2} d_{3} d_{4}}\right)$ with $d_{i}<0,(i=1,2,3), d_{4}>0$ and such that

$$
\left(\frac{d_{1}}{p_{2}}\right)=\left(\frac{d_{2}}{p_{3}}\right)=\left(\frac{d_{3}}{p_{1}}\right)=\left(\frac{d_{1}}{p_{4}}\right)=-1, \quad\left(\frac{d_{4}}{p_{2}}\right)=\left(\frac{d_{4}}{p_{3}}\right)=+1 .
$$

Here is a list of the 2-class numbers of the quadratic subfields of $L=k^{1}$. Along with $h_{2}(k)=8$, we have

$$
\begin{gathered}
h_{2}\left(d_{j}\right)=h_{2}\left(d_{1} d_{2}\right)=h_{2}\left(d_{2} d_{3}\right)=h_{2}\left(d_{1} d_{3}\right)=1, \quad(j=1,2,3,4) \\
h_{2}\left(d_{1} d_{4}\right)=h_{2}\left(d_{1} d_{2} d_{4}\right)=h_{2}\left(d_{1} d_{3} d_{4}\right)=2, \quad h_{2}\left(d_{1} d_{2} d_{3}\right)=4, \\
h_{2}\left(d_{3} d_{4}\right)=2^{l}, \quad h_{2}\left(d_{2} d_{3} d_{4}\right)=2^{m}, \quad h_{2}\left(d_{2} d_{4}\right)=2^{n} \quad(l, m, n \geq 2) .
\end{gathered}
$$

Let $K=L^{+}=\mathbb{Q}\left(\sqrt{d_{1} d_{2}}, \sqrt{d_{1} d_{3}}, \sqrt{d_{4}}\right)$, the maximal real subfield of $L$. Then $q(L / \mathbb{Q})=Q(L / K) q(K / \mathbb{Q})$ by [Benjamin et al. 2003, Proposition 1]. But by [Lemmermeyer 1995], $Q(L / K)=2$. In fact, if $w_{L}\left(=\# W_{L}\right) \equiv 2 \bmod 4$, then $L=K\left(\sqrt{d_{1}}\right)$ and $\left(p_{1}\right)=(\pi)^{2}$ in $Q\left(\sqrt{d_{1} d_{2}}\right)$ since $p_{1}$ ramifies and the field has odd class number. But then $d_{1} \mathbb{O}_{K}=\left(\pi \mathbb{O}_{K}\right)^{2}$, and part (i)2(a) of [Lemmermeyer 1995, Theorem 1] implies $Q(L / K)=2$. If instead $w_{L} \equiv 4 \bmod 8$, then $20_{K}=(1+i)^{2} \widehat{O}_{K}$, whence part (ii)2(a) of the same theorem shows again that $Q(L / K)=2$.

Now we compute $q(K / \mathbb{Q})$. To this end, consider the quadratic number field $k_{0}=$ $\mathbb{Q}\left(\sqrt{d_{2} d_{3} d_{4}}\right)$ with 2-class group $\mathrm{Cl}_{2}\left(k_{0}\right)=\left(2^{m}\right)$ and fundamental unit $\varepsilon_{234}$. Then $K / k_{0}$ is a $V_{4}$-extension with the quadratic subextensions $K_{1}=k_{0}\left(\sqrt{d_{1} d_{2}}\right), K_{2}=$ $k_{0}\left(\sqrt{d_{1} d_{3}}\right), K_{3}=k_{0}\left(\sqrt{d_{2} d_{3}}\right)$. Let $\varepsilon_{i j}$ denote the fundamental unit of $\mathbb{Q}\left(\sqrt{d_{i} d_{j}}\right)$ for $1 \leq i<j \leq 3$. We shall determine $\mathrm{Cl}_{2}\left(K_{1}\right)$ and $q\left(K_{1} / \mathbb{Q}\right)$. Since $k_{0}$ has cyclic 2-class group of type $\left(2^{m}\right)$ and since $K_{1} / k_{0}$ is ramified, its class group contains $\left(2^{m}\right)$ as a subgroup. If we can show that $h_{2}\left(K_{1}\right)=2^{m}$, then $\mathrm{Cl}_{2}\left(K_{1}\right) \simeq\left(2^{m}\right)$; since $K / K_{1}$ is unramified, it would then follow that $\mathrm{Cl}_{2}(K) \simeq\left(2^{m-1}\right)$. Applying Kuroda's class number formula to $K / \mathbb{Q}$ would then give $q(K / \mathbb{Q})=2^{6}$, and this in turn implies $q(L / \mathbb{Q})=2^{7}$ and $h_{2}(L)=2^{l+m+n-1}$.

For computing the 2-class number of $K_{1}$ we use Kuroda's formula

$$
h_{2}\left(K_{1}\right)=\frac{1}{4} q\left(K_{1} / \mathbb{Q}\right) h_{2}\left(d_{1} d_{2}\right) h_{2}\left(d_{1} d_{3} d_{4}\right) h_{2}\left(d_{2} d_{3} d_{4}\right)=q\left(K_{1} / \mathbb{Q}\right) 2^{m-1} .
$$

It suffices to show that $q\left(K_{1} / \mathbb{Q}\right) \leq 2$ (which implies $q\left(K_{1} / \mathbb{Q}\right)=2$ by the argument above).

We consider two cases: $d_{k}:=\operatorname{disc} k \not \equiv 4 \bmod 8$ and $d_{k} \equiv 4 \bmod 8$. Assume $d_{k} \not \equiv$ $4 \bmod 8$. The prime ideal above $d_{1}$ in $\mathbb{Q}\left(\sqrt{d_{1} d_{2}}\right)$ is principal; hence $X^{2}-d_{1} d_{2} y^{2}=$ 


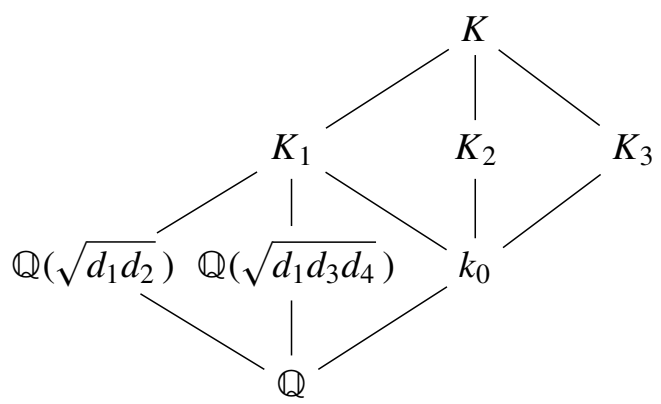

Figure 1. Some subfields of $K / \mathbb{Q}$.

$\pm 4 d_{1}$ is solvable, and so is $d_{1} x^{2}-d_{2} y^{2}=-4$ (the minus sign must occur since $\left.\left(d_{1} / p_{2}\right)=-1\right)$. Then $\eta=\frac{1}{2}\left(x \sqrt{d_{1}}+y \sqrt{d_{2}}\right)$ is a unit in $F=\mathbb{Q}\left(\sqrt{d_{1}}, \sqrt{d_{2}}\right)$; note that $\eta^{2}<0$ in $\mathbb{Q}\left(\sqrt{d_{1} d_{2}}\right)$ since otherwise $\eta \in \mathbb{R} \cap F=\mathbb{Q}\left(\sqrt{d_{1} d_{2}}\right)$. Therefore $\eta^{2}=-\varepsilon_{12}^{u}$; notice that $u$ is odd since otherwise $\sqrt{-1} \in \mathbb{Q}\left(\sqrt{d_{1}}, \sqrt{d_{2}}\right)$, a contradiction. Thus $-d_{1} \varepsilon_{12}=\left(\sqrt{d_{1}} \eta \varepsilon_{12}^{(1-u) / 2}\right)^{2}$ is a square in $\mathbb{Q}\left(\sqrt{d_{1} d_{2}}\right)$.

Next consider $\mathbb{Q}\left(\sqrt{d_{1} d_{3} d_{4}}\right)$, along with the diophantine equations

$$
d_{1} x^{2}-d_{3} d_{4} y^{2}= \pm 4, \quad d_{3} x^{2}-d_{1} d_{4} y^{2}= \pm 4, \quad d_{4} x^{2}-d_{1} d_{3} y^{2}= \pm 4,
$$

which are solvable if the prime above $d_{1}, d_{3}, d_{4}$, respectively, is principal. The first implies $\left(d_{1} / p_{4}\right)=+1$, which contradicts the assumptions. The last implies $\left(d_{4} / p_{1}\right)=\left(d_{4} / p_{3}\right)$, which also leads to a contradiction. Thus the second equation must have a solution, and reduction mod $p_{3}$ shows that we must have $d_{3} x^{2}-d_{1} d_{4} y^{2}=-4$. Thus $-d_{3} \varepsilon_{134}$ is a square in $\mathbb{Q}\left(\sqrt{d_{1} d_{3} d_{4}}\right)$. Hence none of $\varepsilon_{12}, \varepsilon_{134}, \varepsilon_{12} \varepsilon_{134}$ can be squares in $K_{1}$. Therefore $q\left(K_{1} / \mathbb{Q}\right) \leq 2$, as desired.

Now suppose $d_{k} \equiv 4 \bmod 8$. Then our assumptions imply that $d_{3}=-4$ or $d_{2}=-4$. First assume that $d_{3}=-4$. Then the argument above shows that $\varepsilon_{12}=$ $p_{1} \kappa^{2}$ for some $\kappa \in \mathbb{Q}\left(\sqrt{d_{1} d_{2}}\right)$. Now consider $\mathbb{Q}\left(\sqrt{d_{1} d_{3} d_{4}}\right)=\mathbb{Q}\left(\sqrt{p_{1} p_{4}}\right)$. Then by genus theory ([Lemmermeyer 2000, page 76]) there is a principal ideal $(\alpha)$ in $\mathbb{Q}\left(\sqrt{p_{1} p_{4}}\right)$ different from $(1)$ and $\left(\sqrt{p_{1} p_{4}}\right)$ which is a product of distinct ramified prime ideals. We now consider the possibilities. First notice that the prime ideals above $p_{1}$ and $p_{4}$ are not principal, since otherwise $p_{1} x^{2}-p_{4} y^{2}= \pm 1$ is solvable which cannot happen. Now assume that the prime ideal above 2 is principal, equal to say $(\pi)$ with $\pi=x+y \sqrt{p_{1} p_{4}}$, for some $x, y \in \mathbb{N}$. Then $\pi^{2} / 2=\mu$ a positive unit in $\mathbb{Q}\left(\sqrt{p_{1} p_{4}}\right)$. Clearly $\mu$ is not a square in $\mathbb{Q}\left(\sqrt{p_{1} p_{4}}\right)$ since otherwise $\sqrt{2} \in$ $\mathbb{Q}\left(\sqrt{p_{1} p_{4}}\right)$, a contradiction. Hence $\varepsilon_{134}=2 \kappa^{2}$, for some $\kappa \in \mathbb{Q}\left(\sqrt{p_{1} p_{4}}\right)$. Similarly $\varepsilon_{134}$ could be of the form $2 p_{1} \kappa^{2}$ or $2 p_{4} \kappa^{2}$. But in all of these cases we see that none of $\varepsilon_{12}, \varepsilon_{134}, \varepsilon_{12} \varepsilon_{134}$ can be squares in $K_{1}$. Once again we have $q\left(K_{1} / \mathbb{Q}\right) \leq 2$. 
Finally, suppose $d_{2}=-4$. The argument above shows $\varepsilon_{134}=p_{3} \kappa^{2}$, for some $\kappa \in \mathbb{Q}\left(\sqrt{d_{1} d_{3} d_{4}}\right)$. Now consider $\mathbb{Q}\left(\sqrt{d_{1} d_{2}}\right)=\mathbb{Q}\left(\sqrt{p_{1}}\right)$. Then arguing as above we see $\varepsilon_{12}=2 \kappa^{2}$ or $\varepsilon_{12}=2 p_{1} \kappa^{2}$ for some $\kappa \in \mathbb{Q}\left(\sqrt{p_{1}}\right)$. But again this implies $q\left(K_{1} / \mathbb{Q}\right) \leq 2$; whence the result is established.

Now we come to the case where $\mathrm{Cl}_{2}(k) \simeq(2,2,2)$ but with disc $k$ divisible by three positive prime discriminants, say disc $k=d_{1} d_{2} d_{3} d_{4}$ with $d_{i}>0$ for $i=1,2,3$ and $d_{4}<0$. Our results in this case will be far less complete since our knowledge of $\mathrm{Gal}\left(k^{2} / k\right)$ is much more spotty. But we now simplify things somewhat by reducing to the maximal real subfield of $k^{1}$. To this end, from now on, let $L=k^{1}$ and $K=L^{+}$ the maximal real subfield of $L$. But then

$$
q(L / \mathbb{Q})=q(K / \mathbb{Q}),
$$

because $q(L / \mathbb{Q})=Q(L / K) q(K / \mathbb{Q})$ (by [Benjamin et al. 2003, Proposition 1], for example). By [Lemmermeyer 1995, Theorem 1] we get $Q(L / K)=1$ since $L=K\left(\sqrt{d_{4}}\right)$ is essentially ramified if $d_{4} \neq-4$ and $20_{K}$ is not an ideal square when $d_{4}=-4$.

Now we need only consider $K=\mathbb{Q}\left(\sqrt{p_{1}}, \sqrt{p_{2}}, \sqrt{p_{3}}\right)$ where $p_{i}$ are the rational primes dividing $d_{i}$. We set up the following notation. Let $k_{0}=\mathbb{Q}\left(\sqrt{p_{1} p_{2} p_{3}}\right)$. Let $K_{i}=k_{0}\left(\sqrt{p_{i}}\right)$ for $i=1,2,3$ and let $k_{i}$ be the quadratic subfield of $K_{i}$ not equal to $k_{0}$ and $\mathbb{Q}\left(\sqrt{p_{i}}\right)$. (Notice that $k_{i}=\mathbb{Q}\left(\sqrt{\operatorname{disc} k_{0} / p_{i}}\right)$.) We now let $\varepsilon_{i}$ for $i=0,1,2,3$ be the fundamental unit $>1$ in $k_{i}$ and $N \varepsilon_{i}$ the norm from $k_{i}$ to $\mathbb{Q}$; also let $\varepsilon_{p_{i}}$ be the fundamental unit in $\mathbb{Q}\left(\sqrt{p_{i}}\right)$. Finally let $H_{i}=\operatorname{Gal}\left(k_{0}^{2} / K_{i}\right)$.

Now we assume that $k_{0}$ is a particular type of field. Namely, assume that $\mathrm{Cl}_{2}\left(k_{0}\right) \simeq(2,2)$. This assumption implies that $G=\mathrm{Gal}\left(k_{0}^{2} / k_{0}\right)$ is one of the following types: abelian, quaternion, dihedral, semidihedral. Moreover notice that in this case $k_{0}^{1}=K=\mathbb{Q}\left(\sqrt{p_{1}}, \sqrt{p_{2}}, \sqrt{p_{3}}\right)$ since $K / k_{0}$ is unramified and $h_{2}\left(k_{0}\right)=4$. Without loss of generality we now pick $K_{1}$ above so that $H_{1}$ is cyclic. We are in a position to state and prove the following (rather technical) theorem.

Theorem 5. Let $k$ be a complex quadratic field with $\mathrm{Cl}_{2}(k) \simeq(2,2,2)$ and with disc $k=d_{1} d_{2} d_{3} d_{4}$ where $d_{i}$ are distinct prime discriminants divisible by primes $p_{i}$ and $d_{1}, d_{2}, d_{3}$ are positive. With the notation above, assume that $\mathrm{Cl}_{2}\left(k_{0}\right) \simeq(2,2)$. Then $q=q\left(k^{1} / \mathbb{Q}\right)$ takes on the two values $2^{4}$ and $2^{5}$ as follows:

- If $G$ is abelian, then $q=2^{4}$.

- If $G$ is nonabelian, then $N \varepsilon_{0}=+1$ implies $q=\left\{\begin{array}{l}2^{4} \text { if } N \varepsilon_{1}=-1, \\ 2^{5} \text { otherwise, }\end{array}\right\}$, while

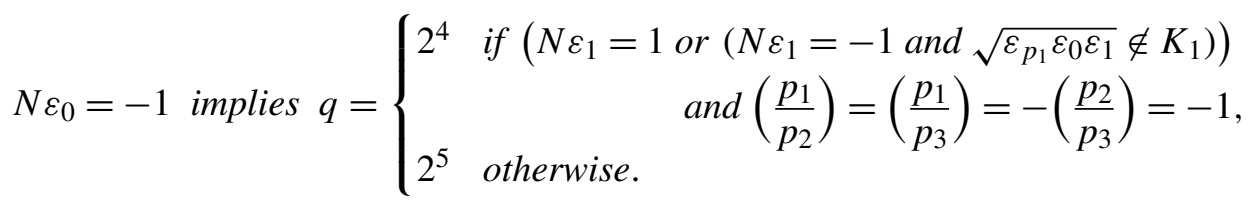


Proof. Since $H_{1}$ is cyclic (and so in particular abelian), we have $k_{0}^{2}=K_{1}^{1}$. Thus $h_{2}\left(K_{1}\right)=\left[k_{0}^{2}: k_{0}^{1}\right]\left[k_{0}^{1}: K_{1}\right]=2 h_{2}\left(k_{0}^{1}\right)$, and hence

$$
h_{2}\left(k_{0}^{1}\right)=\frac{1}{2} h_{2}\left(K_{1}\right) \text {. }
$$

Next notice by Kuroda's class number formula that

$$
h_{2}\left(k_{0}^{1}\right)=\frac{1}{2^{7}} q h_{2}\left(k_{1}\right) h_{2}\left(k_{2}\right) h_{2}\left(k_{3}\right),
$$

where we have used $v=9$ and $h_{2}\left(k_{0}\right)=4$. Now Kuroda's class number formula for $K_{i}$ yields

$(* * *)$

$$
h_{2}\left(K_{i}\right)=\frac{1}{4} q_{i} h_{2}\left(p_{i}\right) h_{2}\left(k_{i}\right) h_{2}\left(k_{0}\right)=q_{i} h_{2}\left(k_{i}\right) .
$$

But then $(*),(* *),(* * *)$ imply

$$
\frac{1}{2} q_{1} h_{2}\left(k_{1}\right)=\frac{1}{2^{7}} q h_{2}\left(k_{1}\right) \frac{h_{2}\left(K_{2}\right)}{q_{2}} \frac{h_{2}\left(K_{3}\right)}{q_{3}},
$$

and therefore

$$
q=\frac{2^{6} q_{1} q_{2} q_{3}}{h_{2}\left(K_{2}\right) h_{2}\left(K_{3}\right)} .
$$

Suppose first of all that $G$ is abelian. Then $k_{0}^{2}=k_{0}^{1}$ and so $h_{2}\left(K_{i}\right)=2$. Thus $q=2^{4} q_{1} q_{2} q_{3}$. But $h_{2}\left(k_{i}\right) \equiv 0 \bmod 2$, since the $d_{j}>0$ for $j=1,2,3$. So $(* * *)$ implies that $h_{2}\left(K_{i}\right)=2=q_{i} h_{2}\left(k_{i}\right)$ and this in turn yields $h_{2}\left(k_{i}\right)=2$ and $q_{i}=1$, for $i=1,2,3$. Thus when $G$ is abelian, $q=2^{4}$.

Now assume that $G$ is not abelian. Then for $G \simeq H_{8}$, the quaternion group of order 8 , or $G \simeq D_{4}$, the dihedral group of order $8, H_{i}$ has order 4 for $i=1,2,3$ and so in particular $h_{2}\left(K_{i}\right)=4$. If $G \not \subset H_{8}$ or $D_{4}$, then $H_{2}, H_{3}$ are either dihedral, semidihedral, or quaternion, whence in particular the abelianizations $H_{2}^{a b} \simeq H_{3}^{a b} \simeq$ $(2,2)$ and thus $h_{2}\left(K_{2}\right)=h_{2}\left(K_{3}\right)=4$. Then

$$
q=2^{2} q_{1} q_{2} q_{3}
$$

Case 1. Assume $N \varepsilon_{0}=1$. We now compute the $q_{i}$ 's. First consider $q_{2}$. By [Couture and Derhem 1992, Theorem 1], $\left(p_{1} / p_{3}\right)=-1$, whence $h_{2}\left(k_{2}\right)\left(=h_{2}\left(p_{1} p_{3}\right)\right)=2$. But $4=h_{2}\left(K_{2}\right)=q_{2} h_{2}\left(k_{2}\right)=2 q_{2}$, so thus

$$
q_{2}=2 \text {. }
$$

Next consider $q_{3}$. Again by [Couture and Derhem 1992, Theorem 1], $\left(p_{2} / p_{1}\right)=$ 1 and $\left(p_{1} / p_{2}\right)_{4}=-\left(p_{2} / p_{1}\right)_{4}$. Since $4=h_{2}\left(K_{3}\right)=q_{3} h_{2}\left(p_{1} p_{2}\right)$, then either $\left(h_{2}\left(p_{1} p_{2}\right)=2 \& q_{3}=2\right)$ or $\left(h_{2}\left(p_{1} p_{2}\right)=4 \quad \& \quad q_{3}=1\right)$. We claim the latter does not hold. For, first by $(\alpha)$ on page 318 of [Kaplan 1976], $\mathrm{Cl}_{2}^{+}\left(k_{3}\right) \simeq$ (4). 
Hence if the latter holds, then $N \varepsilon_{3}=-1$, which is not possible by [Kaplan 1976, Corollary 1]. Hence

$$
q_{3}=2 \text {. }
$$

Finally consider $q_{1}$. First assume $N \varepsilon_{1}=-1$. Then by [Kubota 1956] the only possible square root of a nonsquare unit in $K_{1}$ would be $\sqrt{\varepsilon_{0}}$ since the others have negative norm. Now applying [Benjamin et al. 1998, Proposition 3], for example, we see $k_{0}\left(\sqrt{\varepsilon_{0}}\right)=k_{0}(\sqrt{\delta})$, where by genus theory $\delta \mid p_{1} p_{2} p_{3}$ (but $\neq$ ) and $\chi_{j}(\delta)=1$ for all genus characters of $k_{0}$. But then since $\left(p_{1} / p_{2}\right)=\left(p_{3} / p_{2}\right)=1, p_{2}$ is trivial for all the genus characters and no other $p_{i}$ has this property. Thus we may assume $\delta=p_{2}$ which is not a square in $K_{1}$. Thus

$$
N \varepsilon_{1}=-1 \quad \text { implies } \quad q_{1}=1 .
$$

Now assume $N \varepsilon_{1}=+1$. Then $\delta_{k_{0}}=p_{2}$ again and $\delta_{k_{1}}=p_{2}$ so that $\varepsilon_{0} \varepsilon_{1}$ is a square in $K_{1}$ this time; again see [Kubota 1956]. Hence

$$
N \varepsilon_{1}=+1 \quad \text { implies } \quad q_{1}=2 .
$$

Therefore, for $N \varepsilon_{0}=1, q=2^{4}$ if $N \varepsilon_{1}=-1$ and $q=2^{5}$ if $N \varepsilon_{1}=+1$.

Case 2. Assume $N \varepsilon_{0}=-1$. Since $\mathrm{Cl}_{2}\left(k_{0}\right)=\mathrm{Cl}_{2}^{+}\left(k_{0}\right)$ is elementary, the RédeiReichardt conditions [1933] imply that

a) $\left(\frac{p_{i}}{p_{j}}\right)=-1, \quad$ for all $i \neq j, \quad$ or $\left.\quad b\right) \quad\left(\frac{p_{1}}{p_{2}}\right)=\left(\frac{p_{1}}{p_{3}}\right)=-\left(\frac{p_{2}}{p_{3}}\right)=-1$.

First consider $a)$. Then $\left(p_{i} p_{j} / p_{\ell}\right)=1$ for all distinct $i, j, \ell=1,2,3$. By [Couture and Derhem 1992, Theorem 2], $G \simeq(2,2)$ or $H_{8}$. Hence in the present situation $G \simeq H_{8}$. Thus as noted above the order of $H_{i}$ is 4 whence $h_{2}\left(K_{i}\right)=4$ so that $4=h_{2}\left(K_{i}\right)=q_{i} h_{2}\left(k_{i}\right)$, for $i=1,2,3$. But $\left(p_{i} / p_{j}\right)=-1$ implies $h_{2}\left(k_{i}\right)=2$. Therefore, $q_{i}=2$ for $i=1,2,3$ so $q=2^{5}$.

Next consider $b$ ). As immediately above, $q_{2}=q_{3}=2$. Now consider $q_{1}$. If $N \varepsilon_{1}=+1$, then arguing as above shows $q_{1}=1$. If $N \varepsilon_{1}=-1$, so that the norms of $\varepsilon_{p_{1}}, \varepsilon_{1}, \varepsilon_{0}$ are negative, then $q_{1}=1$ if $\sqrt{\varepsilon_{p_{1}} \varepsilon_{1} \varepsilon_{0}} \notin K_{1}$, and $q_{1}=2$ otherwise.

This establishes the theorem.

As a corollary to this theorem, we see that the structure $G=\operatorname{Gal}\left(k_{0}^{2} / k_{0}\right)$ determines $q\left(k^{1} / \mathbb{Q}\right)$ :

Corollary 6. Let $k_{0}$ satisfy all the conditions in Theorem 5. For $G=\operatorname{Gal}\left(k_{0}^{2} / k_{0}\right)$,

$$
q\left(k^{1} / \mathbb{Q}\right)= \begin{cases}2^{4} & \text { if } G \text { is abelian or dihedral, } \\ 2^{5} & \text { if } G \text { is semidihedral or quaternion } .\end{cases}
$$


Proof. This follows immediately by Theorem 1 of [Couture and Derhem 1992] and a stronger form of part of Theorem 2 of the same paper, as found in [Lemmermeyer 1994a]. The main change in Theorem 2 is the following: with the notation above Theorem 5 suppose $N \varepsilon_{0}=N \varepsilon_{1}=-1$. If $\sqrt{\varepsilon_{p_{1}} \varepsilon_{1} \varepsilon_{0}} \in K_{1}$, then $G$ is quaternion (of order 8 or larger). If $\sqrt{\varepsilon_{p_{1}} \varepsilon_{1} \varepsilon_{0}} \notin K_{1}$, then $G$ is dihedral.

The previous theorem is a special case of the following proposition:

Proposition 7. Let $k$ be a complex quadratic field with $\mathrm{Cl}_{2}(k) \simeq(2,2,2)$ and with disc $k=d_{1} d_{2} d_{3} d_{4}$ where $d_{i}$ are distinct prime discriminants divisible by primes $p_{i}$ and $d_{1}, d_{2}, d_{3}$ are positive. With the notation above, assume that $k_{0}^{1}=k_{0}^{2}$. Then $q=q\left(k^{1} / \mathbb{Q}\right)=2^{4}$.

Proof. Recall that $k_{0}=\mathbb{Q}\left(\sqrt{p_{1} p_{2} p_{3}}\right), K=\mathbb{Q}\left(\sqrt{p_{1}}, \sqrt{p_{2}}, \sqrt{p_{3}}\right)$, and that $q=$ $q(K / \mathbb{Q})$. Then Kuroda's class number formula yields

$$
h_{2}(K)=\frac{1}{2^{9}} q h_{2}\left(k_{0}\right) h_{2}\left(k_{1}\right) h_{2}\left(k_{2}\right) h_{2}\left(k_{3}\right)
$$

(again, refer to the notation before the previous theorem). Now since $k_{0} \subseteq K \subseteq k_{0}^{1}$, we see $k_{0}^{1} \subseteq K^{1} \subseteq k_{0}^{2}$; but since by assumption $k_{0}^{1}=k_{0}^{2}$, we have $K^{1}=k_{0}^{1}$. Hence

$$
h_{2}(K)=\left[K^{1}: K\right]=\left[k_{0}^{1}: K\right]=\frac{1}{4}\left[k_{0}^{1}: k_{0}\right]=\frac{1}{4} h_{2}\left(k_{0}\right) .
$$

Similarly $K_{i}^{1}=k_{0}^{1}$, for $i=1,2,3$, whence

$$
h_{2}\left(K_{i}\right)=\frac{1}{2} h_{2}\left(k_{0}\right) \text {. }
$$

On the other hand Kuroda's class number formula again yields

$$
h_{2}\left(K_{i}\right)=\frac{1}{4} q_{i} h_{2}\left(k_{0}\right) h_{2}\left(k_{i}\right) .
$$

All this implies

$$
\frac{1}{2} h_{2}\left(k_{0}\right)=\frac{1}{4} q_{i} h_{2}\left(k_{0}\right) h_{2}\left(k_{i}\right)
$$

so that $2=q_{i} h_{2}\left(k_{i}\right)$. But then since $2 \mid h_{2}\left(k_{i}\right)$, we must have $h_{2}\left(k_{i}\right)=2$. But then from above we have

$$
\frac{1}{4} h_{2}\left(k_{0}\right)=h_{2}(K)=\frac{1}{2^{9}} q h_{2}\left(k_{0}\right) h_{2}\left(k_{1}\right) h_{2}\left(k_{2}\right) h_{2}\left(k_{3}\right)=\frac{1}{2^{6}} q h_{2}\left(k_{0}\right) .
$$

Therefore by solving for $q$, we obtain

$$
q=2^{4}
$$




\section{Examples}

We now give numerical examples illustrating Theorem 5 with $q=2^{4}$ and $q=2^{5}$.

Example 1. Let $k_{0}=\mathbb{Q}(\sqrt{2405})=\mathbb{Q}(\sqrt{5 \cdot 13 \cdot 37})$ and $K=\mathbb{Q}(\sqrt{5}, \sqrt{13}, \sqrt{37})$. By [Rédei and Reichardt 1933] or [Kaplan 1976] we see that $\mathrm{Cl}_{2}\left(k_{0}\right) \simeq(2,2)$. Moreover, we have $N \varepsilon_{0}=-1$ and $(13 / 5)=(37 / 5)=(37 / 13)=-1$. Thus by [Couture and Derhem 1992, Theorem 2], $\operatorname{Gal}\left(k_{0}^{2} / k_{0}\right) \simeq H_{8}$ or $(2,2)$; but [Benjamin et al. 1998, Theorem 1] then shows that $\operatorname{Gal}\left(k_{0}^{2} / k_{0}\right) \simeq H_{8}$. Finally Theorem 4 above shows $q=2^{5}$.

Example 2. Consider $k_{0}=\mathbb{Q}(\sqrt{290})=\mathbb{Q}(\sqrt{2 \cdot 5 \cdot 29})$; see the examples in [Couture and Derhem 1992]. Let $K=\mathbb{Q}(\sqrt{2}, \sqrt{5}, \sqrt{29})$. By [Rédei and Reichardt 1933] or [Kaplan 1976] or even [Couture and Derhem 1992], we see that $\mathrm{Cl}_{2}\left(k_{0}\right) \simeq(2,2)$. Moreover, we have $N \varepsilon_{0}=-1$, where $\varepsilon_{0}=17+\sqrt{290}$ is the fundamental unit of $k_{0}$; and $(2 / 5)=(2 / 29)=-(29 / 5)=-1$. Now by genus theory $K_{1}=\mathbb{Q}(\sqrt{5 \cdot 29}, \sqrt{2})$ (notation as in above). Also $N \varepsilon_{1}=-1$ where $\varepsilon_{1}=12+\sqrt{145}$ is the fundamental unit of $\mathbb{Q}(\sqrt{5 \cdot 29})$. Finally, $\varepsilon_{2}=1+\sqrt{2}$. By the techniques described in [Kubota 1956] we see that $\varepsilon_{0} \varepsilon_{1} \varepsilon_{2}$ is not a square in $K_{1}$. Theorem 5 above then shows $q=2^{4}$. Furthermore, [Couture and Derhem 1992, Theorem 2] and PARI show $\operatorname{Gal}\left(k_{0}^{2} / k_{0}\right) \simeq D_{4}$.

\section{References}

[Benjamin et al. 1997] E. Benjamin, F. Lemmermeyer, and C. Snyder, "Imaginary quadratic fields $k$ with cyclic $\mathrm{Cl}_{2}\left(k^{1}\right)$ ”, J. Number Theory 67:2 (1997), 229-245. MR 99a:11126 Zbl 0919.11074

[Benjamin et al. 1998] E. Benjamin, F. Lemmermeyer, and C. Snyder, "Real quadratic fields with abelian 2-class field tower", J. Number Theory 73:2 (1998), 182-194. MR 2000c:11179 Zbl 0919. 11073

[Benjamin et al. 2001] E. Benjamin, F. Lemmermeyer, and C. Snyder, "Imaginary quadratic fields $k$ with $\mathrm{Cl}_{2}(k) \simeq\left(2,2^{m}\right)$ and rank $\mathrm{Cl}_{2}\left(k^{1}\right)=2$ ", Pacific J. Math. 198 (2001), 15-31. MR 2002c:11147 Zbl 1063.11038

[Benjamin et al. 2003] E. Benjamin, F. Lemmermeyer, and C. Snyder, "Imaginary quadratic fields with $\mathrm{Cl}_{2}(k) \cong(2,2,2)$ ", J. Number Theory 103:1 (2003), 38-70. MR 2004f:11124 Zbl 1045.11077

[Couture and Derhem 1992] R. Couture and A. Derhem, "Un problème de capitulation", C. R. Acad. Sci. Paris Sér. I Math. 314:11 (1992), 785-788. MR 93c:11100 Zbl 0778.11059

[Dirichlet 1842] G. L. Dirichlet, "Recherches sur les formes quadratiques à coëfficients et à indéterminées complexes", J. reine angew. Math. 24 (1842), 291-371.

[Hall 1933] P. Hall, "A contribution to the theory of groups of prime-power order", Proc. London Math. Soc. 36 (1933), 29-95. Zbl 0007.29102 JFM 59.0147.02

[Hall and Senior 1964] M. Hall, Jr. and J. K. Senior, The groups of order $2^{n}(n \leq 6)$, Macmillan, New York, 1964. MR 29 \#5889 Zbl 0192.11701

[Hasse 1985] H. Hasse, Über die Klassenzahl abelscher Zahlkörper, Springer-Verlag, Berlin, 1985. MR 87j:11122a Zbl 0668.12004 
[Herglotz 1922] G. Herglotz, "Über einen Dirichletschen Satz”, Math. Z. 12:1 (1922), 255-261. MR MR1544516 JFM 48.0170.01

[Kaplan 1976] P. Kaplan, "Sur le 2-groupe des classes d'idéaux des corps quadratiques", J. Reine Angew. Math. 283/284 (1976), 313-363. MR 53 \#8009 Zbl 0337.12003

[Kubota 1953] T. Kubota, "Über die Beziehung der Klassenzahlen der Unterkörper des bizyklischen biquadratischen Zahlkörpers", Nagoya Math. J. 6 (1953), 119-127. MR 15,605e Zbl 0053.21902

[Kubota 1956] T. Kubota, "Über den bizyklischen biquadratischen Zahlkörper”, Nagoya Math. J. 10 (1956), 65-85. MR 18,643e Zbl 0074.03001

[Kuroda 1950] S. Kuroda, "Über die Klassenzahlen algebraischer Zahlkörper”, Nagoya Math. J. 1 (1950), 1-10. MR 12,593a Zbl 0037.16101

[Lemmermeyer 1994a] F. Lemmermeyer, Die Konstruktion von Klassenkörpern, Doctoral Dissertation, Universität Heidelberg, 1994.

[Lemmermeyer 1994b] F. Lemmermeyer, “Kuroda's class number formula”, Acta Arith. 66:3 (1994), 245-260. MR 95f:11090 Zbl 0807.11052

[Lemmermeyer 1995] F. Lemmermeyer, "Ideal class groups of cyclotomic number fields, I", Acta Arith. 72:4 (1995), 347-359. MR 96h:11111 Zbl 0837.11059

[Lemmermeyer 2000] F. Lemmermeyer, Reciprocity laws: from Euler to Eisenstein, Springer, Berlin, 2000. MR 2001i:11009 Zbl 0949.11002

[Rédei and Reichardt 1933] L. Rédei and H. Reichardt, "Die Anzahl der durch 4 teilbaren Invarianten der Klassengruppe eines beliebigen quadratischen Zahlkörpers", J. Reine Angew. Math. 170 (1933), 69-74. Zbl 0007.39602

[Wada 1966] H. Wada, "On the class number and the unit group of certain algebraic number fields", J. Fac. Sci. Univ. Tokyo Sect. I 13 (1966), 201-209. MR 35 \#5414 Zbl 0158.30103

Received August 14, 2005.

ELLIOT BENJAMIN

DEPARTMENT OF MATHEMATICS AND STATistics

UNIVERSITY OF MAINE

ORONO, MAINE 04469

UNITED STATES

ben496@prexar.com

FRANZ LEMMERMEYER

DEPARTMENT OF MATHEMATICS

BILKENT UNIVERSITY

06800 BILKENT, ANKARA

TURKEY

franz@fen.bilkent.edu.tr

CHIP SNYDER

DEPARTMENT OF MATHEMATICS AND StATistics

UNIVERSITY OF MAINE

ORONO, MAINE 04469

UNited STATES

snyder@math.umaine.edu 